\title{
HABILIDADES BLANDAS Y TÉCNICAS EN LA PROFESIÓN INGENIERIL EN COSTA RICA. UN ABORDAJE DE ENFOQUES EN TECNOLOGÍAS
}

Soft and technical abilities in the engineering profession in Costa Rica. Technologies focus approach 


\section{RESUMEN}

El artículo aborda los aspectos teóricos y prácticos referentes a la existencia y aplicación de las habilidades técnicas, o bien entendidas como duras, así como las llamadas blandas, en el campo de la ingeniería, realizando un análisis basado en datos de campo a una muestra de 132 ingenieros activos en Costa Rica, en sus respectivos campos. El abordaje de los datos se hace en función del patrón conductual de estos individuos, así como del comportamiento en el ejercicio profesional. Analizadas estas habilidades, se presenta un cruce de información en el cual se precisan las relaciones existentes entre las habilidades de mayor relevancia en la profesión ingenieril y los requerimientos tecnológicos y de aplicaciones informáticas que permiten maximizar las habilidades en cuestión.

Palabras clave: Ingeniería; Habilidades blandas; Habilidades técnicas; Perfiles profesionales; Tecnologías.

\section{ABSTRACT:}

The paper discusses the theoretical and practical aspects related to the existence and application of technical skills, also understood as hard skills, as well as the soft skills, in the field of engineering, performing an analysis based on field data obtained from a sample of 132 active engineers in Costa Rica, in their respective fields. The data approach was done according to the behavioral pattern of these individuals, as well as their behavior in professional performance. Once skills are analyzed, an information crossover is presented, in which the relationships between the most important skills in the engineering profession are related to the technological and computer application requirements that allow to maximize the skills in question.

Key words: Engineering; Soft skills; Technical skills; Professional profiles; Technologies. 


\section{Introducción}

$\mathrm{E}$ 1 artículo presentado detalla los resultados de un estudio de campo realizado a ingenieros activos en su profesión, en el cual se precisan las habilidades de corte técnico, es decir las llamadas habilidades duras, así como las destrezas blandas (Tovar, 2012) de mayor relevancia para el ejercicio de la profesión ingenieril en Costa Rica.

Este estudio es sustentando en los resultados de un instrumento investigativo aplicado a una muestra aleatoria de ingenieros activos en el país, considerando cualquier tipo de ingeniería, detallando el uso de ambos tipos de habilidades. Los datos recolectados son analizados en términos de sus posibles aplicaciones al campo, así como de su ligamen en el uso de diferentes tecnologías que permitan maximizar o potenciar ciertas destrezas encontradas, o bien, en la sugerencia de la incorporación de determinadas aplicaciones tecnológicas que permitan a su vez mejorar aquellas habilidades que no denoten ser las de mayor impacto en las profesiones.

Los resultados referentes a las habilidades encontradas son contrastados contra la literatura analizada, considerando aspectos como el perfil conductual, los patrones de conducta y otras variables de corte psicométrico que permiten profundizar en el análisis de los resultados obtenidos y derivados del estudio de campo. Adicionalmente, se precisan resultados en relación con la discriminación en el ejercicio de la profesión ingenieril en el país, considerando diferentes variables que pudiesen ser como discriminatorias para los ingenieros, detallando a su vez su impacto en el área analizada y sus posibles implicaciones profesionales en el campo.

Finalmente, el artículo termina con un análisis cruzado, donde se valoran algunos requerimientos en materia de tecnologías de información, en referencia directa a las habilidades tanto duras como blandas encontradas en el trabajo, logrando así precisar una especie de análisis multivariado en el cual se detallan algunos enfoques en tecnología que pudiesen beneficiar o potenciar las habilidades precisadas en el trabajo. Cabe señalar que la revisión del documento y la contrastación bibliográfica son utilizadas a lo largo del trabajo, de forma que los resultados de campo puedan ser analizados desde su óptica estrictamente práctica, pero también conceptual. Se detalla el siguiente objetivo para el trabajo:

Precisar las principales habilidades blandas en el ejercicio de la profesión ingenieril en Costa Rica, y su relación aplicativa con la práctica laboral, señalando las relaciones técnicas entre ellas y sus agrupamientos prácticos.

\section{Metodología}

El presente documento y sus correspondientes resultados se derivan de un estudio de campo en el cual se definen como sujetos de investigación a una muestra de ingenieros activos en el ejercicio de su profesión. Para estos efectos la elección se hace de forma probabilística y sin importar la ingeniería específica, ni ninguna otra variable en particular, más sin embargo se solicita como requisito estar activo en su profesión y contar con el grado académico que lo respalde.

Para la escogencia específica de los sujetos de estudio, el instrumento se coloca en una plataforma virtual con accesibilidad por parte de toda la población de estudio, donde cualquier ingeniero activo puede contestar, aplicando un criterio de oportunidad para la elección, señalando que se abre un espacio temporal de dos meses para la recolección de los datos, cerrando este espacio con los primeros 132 
cuestionarios que se reciban.

Definidos estos puntos, se establece una población infinita, esto pues, aunque existente diversos colegios profesionales en el país, se presentan algunas ingenierías que no tienen colegio alguno, y no se observa un registro fehaciente de cuantos ingenieros activos se encuentran ejerciendo su profesión en Costa Rica.

Definido lo anterior se establece una confiabilidad de $95 \%$, que equivale a un valor $\mathrm{Z}$ de 1.96 , definiendo a su vez un error muestral del $4.87 \%$, siendo este el error típico y una homogeneidad de $95 \%$, pues el cuestionario aplicado cuenta con una pregunta filtro (Anderson, Sweeney y Williams, 2008), la cual cumple la función de asegurar que únicamente contesten el instrumento quienes cumplan con el requisito de ser ingeniero activo y ejercicio de la profesión. El cálculo de la muestra del estudio es el siguiente:

$$
\mathrm{n}=\underline{\left[\mathrm{z}^{2}\right.} \frac{\mathrm{p}^{\star}(1-\mathrm{p})}{\mathrm{e}^{2}}
$$

Dónde:

$\mathrm{Z}=$ intervalo de confianza

$\mathrm{p}=$ probabilidad de éxito

$\mathrm{q}=$ probabilidad de fracaso $(1-\mathrm{p})$

$\mathrm{e}=$ error muestral

$132=[1.96 * 2 * 0.95 *(1-0.95)]$

$0.0487^{2}$

\subsection{Instrumento}

Para efectos del presente trabajo se utiliza un cuestionario aplicado a la muestra antes señalada, el cual se ejecuta de forma electrónica, por medio de una plataforma de acceso libre a toda la población de estudio. El documento se desglosa de la siguiente forma

Tabla 1: Desglose del cuestionario aplicado a la muestra de ingenieros en Costa Rica, 2020 -2021

\begin{tabular}{|c|c|c|c|}
\hline Ítem & Naturaleza & Reactivo & Indicador \\
\hline 1 & Politómica cerrada & $\begin{array}{c}\text { Elemento personal de importancia en el } \\
\text { ejercicio de la profesión }\end{array}$ & De razón \\
\hline 2 & Politómica cerrada & $\begin{array}{c}\text { Habilidad blanda primordial en la } \\
\text { ingeniería }\end{array}$ & De razón \\
\hline 3 & $\begin{array}{l}\text { Politómica cerrada, escala tipo } \\
\text { Likert }\end{array}$ & Importancia por habilidad blanda & Ordinal y de razón \\
\hline 4 & $\begin{array}{c}\text { Politómica cerrada, escala tipo } \\
\text { Likert }\end{array}$ & Habilidad técnica más importante & Ordinal y de razón \\
\hline 5 & Cerrada dicotómica & $\begin{array}{c}\text { Discriminación en la profesión inge- } \\
\text { nieril }\end{array}$ & Nominal \\
\hline 6 & Cerrada politómica & $\begin{array}{c}\text { Razón de discriminación en el ejercicio } \\
\text { de la profesión }\end{array}$ & De razón \\
\hline
\end{tabular}

Fuente: Elaboración propia. 


\subsection{Validez del instrumento}

La validez del instrumento se establece por medio del Alfa de Cronbach, herramienta que encuentra sustento en lo descrito por Crisman (2016, p. 83) al definir que: "El alfa de Cronbach es un coeficiente que sirve para medir la fiabilidad de una escala de medida ya que se fundamenta en el promedio de las correlaciones entre los ítems". Señalando que resultados mayores a 0.6 se consideran como aceptables para efectos de validar el instrumento.

Para este indicador se utilizan los ítems que se encuentren en una escala estipulada de forma ordinal, o bien que puede ser convertida a un parámetro numérico. Para este trabajo se utilizan los siguientes ítems:

- Ítem 3 (13 reactivos).

- Ítem 4 (9 reactivos)

- Ítem 5

De esta forma y con base en los indicadores contemplados para el cálculo del coeficiente en cuestión, se precisa que se trabaja con un total de 23 ítems. El cálculo del Alfa de Cronbach se detalla seguidamente:

$\alpha=[\text { Ítems } /(\text { Ítems }-1)]^{*}\left[1-\left(\sum \sigma 2 \mathrm{~N} / \sigma 2 \sum\right.\right.$ Ítems $\left.)\right]$

Dónde:

$a=$ Alfa de Cronbach

Ítems $=$ Cantidad de ítems

$\sum \sigma 2 \mathrm{~N}=$ Sumatoria de la varianza de los sujetos

$\sigma 2 \sum$ Ítems) $=$ Varianza de la sumatoria de los ítems

Aplicando la fórmula para los datos específicos obtenidos en el cuestionario aplicado a los ingenieros de la muestra seleccionada y con base en los ítems antes señalados, se tiene lo siguiente:

$\alpha=[23 /(23-1)]^{*}[1-(27.47 / 96.93]=0.75$

De esta forma logra evidenciarse que los resultados del trabajo son claramente confiables y el instrumento es válido, pues el resultado del coeficiente de Cronbach es mayor a 0.6 (Crisman, 2016).

\subsection{Análisis de datos}

Los datos recopilados son analizados en función de su presentación en gráficos, los cuales son confeccionados de acuerdo con su indicador (Anderson et al., 2008), señalando que las figuras derivadas de ítems nominales y de razón, son precisados de mayor a menor, mientras que aquellos obtenidos de indicadores ordinales, se precisan en forma de barras, respetando el orden de la escala. Los resultados son analizados en función de un enfoque descriptivo y su implicación, precisando su escrutinio en rela- 
ción con la contrastación bibliográfica, esto en aras de comparar empíricamente los datos obtenidos, en función de la teoría existente en el tema

Adicionalmente, se confeccionan diagramaciones de los modelos comparativos de los resultados propios de las habilidades y su aplicación práctica, esto desde una perspectiva cualitativa y analítica (Navarro, 2011), donde se realizan cruces de los indicadores analizados, esto en función de un agrupamiento en categorías, de forma que pueden evidenciarse las relaciones de interés entre las variables. Cabe indicar que la validez de los datos recopilados se precisa por medio del Alfa de Cronbach, tema que a su vez permite brindar la confiabilidad del caso a los resultados y las relaciones señaladas entre ellos.

\section{Habilidades blandas en la ingeniería}

Primeramente, se presentan tanto los elementos propios del perfil conductual profesional de los ingenieros, así como las habilidades blandas de relevancia. Perfil de conducta, que para efectos de este concepto debe entenderse que:

[...] el perfil de conducta que caracteriza a una persona nos permitiría, además, identificar las razones de su comportamiento, que puede variar de unas situaciones a otras, pero que, al mismo tiempo, guarda una lógica y coherencias internas que lo hacen predecible (Bermúdez, Pérez, Ruíz, Suárez y Rueda, 2012, s. p.)

Observando que son variables más asociadas a la personalidad y al ser cognitivo y emocional propios del individuo y conducta individual, pero ligadas completamente al desarrollo de su profesión, así como funciones propias atinentes a su carrera profesional.

Algunos de los elementos de mayor relevancia en términos del perfil conductual profesional de los individuos encuestados son detallados seguidamente. 
Figura 1. Elemento personal de importancia en el ejercicio de la profesión, muestra de ingenieros. 2020-2021



\author{
- Valoración técnica \\ - Conocimiento previo \\ - Racionalidad \\ - Otro \\ - Influencia \\ - Intuición \\ - Emotividad \\ - Impulso
}

Fuente: Elaboración propia con base en trabajo de campo.

En la figura anterior resalta que el perfil de mayor relevancia en los ingenieros es dado más hacia la existencia de variables de corte racional, tales como el conocimiento previo y la valoración técnica, observándose una menor relevancia en las variables asociadas a factores más subjetivos, tales como la emoción y la intuición. Donde puede observarse su ligamen correlacionado con aspectos propios del perfil de la persona y del patrón de conducta propiamente (Solomon, 2013), más que a las especificaciones técnicas de la carrera en sí misma.

Presentados los datos anteriores, proceden a detallarse, presentando los resultados referentes a la habilidad blanda de mayor relevancia en el ejercicio de la profesión ingenieril, definiendo para estos efectos las habilidades o capacidades blandas como: “...destrezas mucho más subjetivas e intangibles. Aspectos como el liderazgo, comunicarse fluidamente tanto individual como grupalmente y manejar con tranquilidad los momentos de presión en el trabajo, entre otros" (Tovar, 2012, p. 36).

Es de interés indicar que las habilidades acá señaladas, aunque no parecen ser propias de las carreras en ingenierías, estas pudiesen ser incentivadas por medio de programas universitarios, pero también incluso, desde la educación básica primaria, y sobre todo la secundaria, pues básicamente, su enseñanza, responde más a temas propios de la persona y a habilidades conductuales de la ingeniería (Sánchez, 2020), mismas que pudiesen gestionarse en programas adjuntos a la educación técnica y general.

\title{
3. Habilidades blandas
}

Seguidamente se detallan los hallazgos referentes a las habilidades blandas referentes al desarrollo de la profesión ingenieril en el país. 
Figura 2. Habilidad blanda primordial en la ingeniería, muestra de ingenieros, 2020-2021.

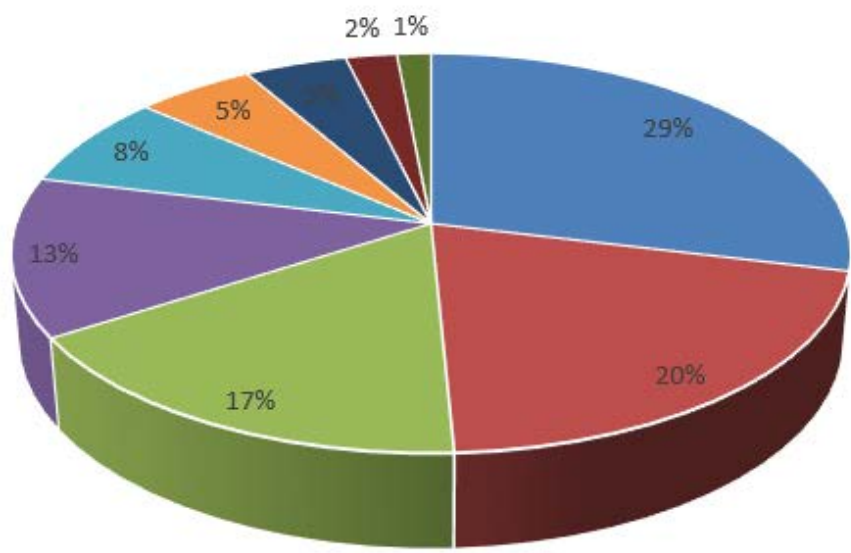

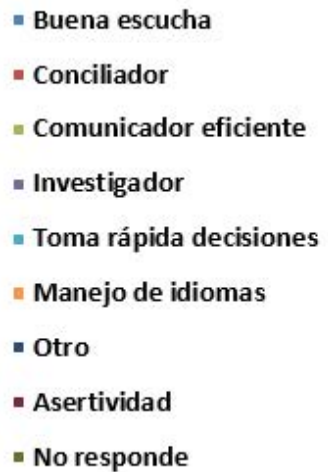

Fuente: Elaboración propia con base en trabajo de campo.

La segunda figura presentada denota la habilidad blanda de mayor relevancia para el ejercicio de la profesión ingenieril en Costa Rica. A este punto debe indicarse que en una segunda aproximación al concepto de las habilidades blandas se entiende por “....quellas que se vuelcan más a la comunicación y desenvolvimiento de las personas en entornos conformados por pares. A esto se la responsabilidad, la honestidad y el compromiso que demanda la resolución de problemas de uso cotidiano" (Luna, Peña, y Iacano, 2018, p. 4).

Primeramente, resalta el concepto de buena escucha, al cual según Martí-Lobo (2015), tiene relación con el tipo de aprendizaje y menciona tener una capacidad de relevancia en la prestación de atención a las situaciones expuestas por otros individuos, también denominada como escucha activa. En segundo lugar, se observa el concepto de conciliador, es decir, la capacidad de negociar y de lograr acuerdos de forma eficaz entre las partes, que denota un cierto grado de inteligencia interpersonal (Galera, 2015). Seguido en tercer lugar por la comunicación eficiente, que claramente señala tener una capacidad atinente a la transmisión de ideas de manera adecuada para con otros.

Con base en lo anterior puede indicarse entonces que el perfil básico, al menos desde un punto de vista de habilidades blandas para efectos del ejercicio profesional de la ingeniería, está basado en la escucha, la conciliación y la capacidad de transmitir de forma clara y precisa las ideas, es decir, puede extrapolarse este comportamiento a un circuito de conducta en el cual se recopila información, se analiza y se comunica, procesos que puede ser de igual forma transformados en un proceso relacionado con sistemas informáticos (Alonso, Martínez y Segovia, 2005). Puede indicarse así, que aplicaciones que potencien este comportamiento, dado en los tres procesos antes señalados, pueden mejorar y maximizar la aplicación de habilidades blandas en la profesión de la ingeniería. Un ejemplo del ligamen de las habilidades blandas para con los sistemas informáticos, es observable en lo expresado por Blanco (2009, p. 21), al señalar que estas: “...son principalmente de tipo personal y social y tienen que ver con habili- 
dades de comunicación, capacidad de trabajar en equipo, comprensión de los sistemas y metodologías de trabajo con tecnologías informáticas".

Pudiendo observar que la gestión de sistemas informáticos, así como el manejo adecuado de este tipo de sistemas, parecen tener una relación directa con las habilidades blandas del ingeniero, lo que denota una relación de interés para el perfil profesional en cuestión, siendo el uso de tecnologías, tanto una capacidad blanda y técnica a la vez. Este proceso de manejo y aplicación de habilidades blandas y su posible análisis desde un enfoque de proceso informático, se detalla en la siguiente figura:

Figura 3: Circuito de comportamiento y operativización en un sistema informático

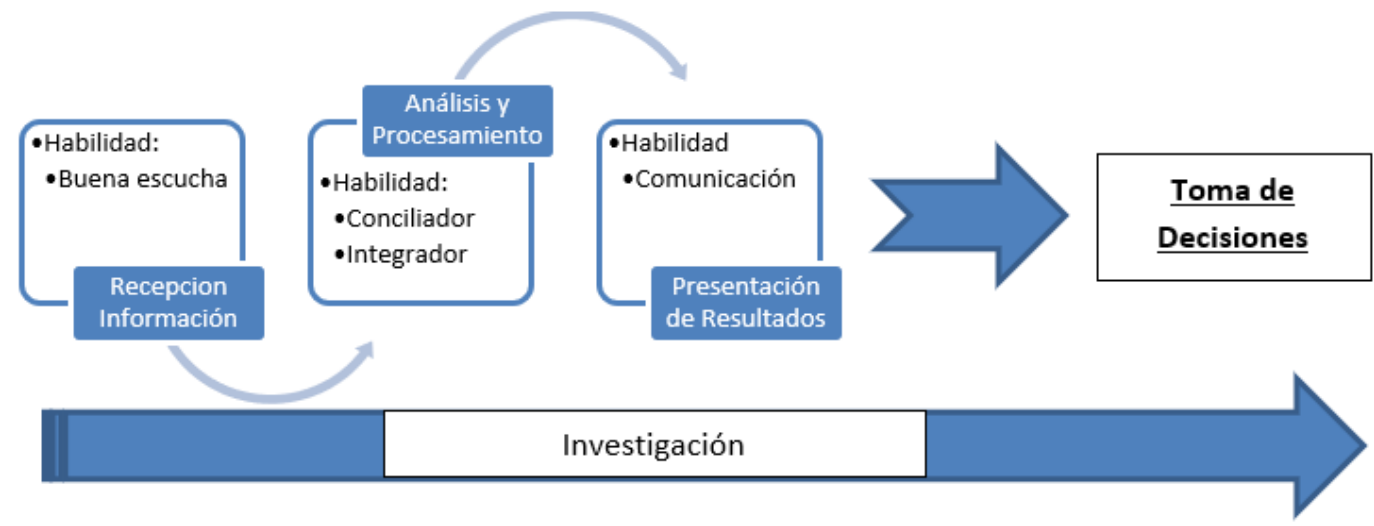

Fuente: Elaboración propia

Puede observarse como las tres habilidades blandas de mayor relevancia son sistematizadas y presentadas desde un punto de vista de un diagrama operativo, en el cual se detalla un posible requerimiento para el desarrollo de habilidades blandas en los ingenieros. A lo que cabe señalar que este diagrama se complementa y viene a detallar sus principales aplicaciones prácticas en las siguientes dos habilidades blandas de mayor importancia, siendo estas la toma de decisiones y la investigación, con esta última jugando un papel de eje transversal en el desarrollo de las habilidades en cuestión.

Es de interés señalar que estas dos últimas habilidades implican, por sí mismas una correcta gestión de la información y de actividades varias ligadas a ello, las que permiten establecer un perfil integral en el profesional, lo que se observa al señalarse que, de acuerdo con Worboys, Lockwood y Kothari (2019, p. 268):

Los ejemplos de las habilidades blandas incluyen: manejo de tiempo; confiabilidad; trabajo en equipo; comunicación interpersonal; lenguaje; deseo de aprender y de recibir capacitación; demostrar integridad y un comportamiento ético; estar motivado y tener una actitud positiva, y analizar críticamente la información.

Puede así indicarse que el comportamiento completo que involucre todo el proceso de gestión de la información, desde su recolección, su procesamiento, sistematización, presentación y finalmente su uso para la eficaz toda de decisiones, denota ser el circuito conductual que maximiza las habilidades blandas en los ingenieros, mismo que debe ser gestionado en este orden en aras de lograr sistematizar posibles aplicaciones y sistemas derivados. La importancia de las habilidades en cuestión se detalla seguidamente. 
Figura 4. Importancia por habilidad blanda, muestra de ingenieros, 2020-2021



Fuente: Elaboración propia con base en trabajo de campo.

En ligamen con lo anterior y en la figura 3 logra observarse la importancia por tipo de habilidad blanda de acuerdo con la muestra de ingenieros analizada. Lo primero que destaca es una aparente relevancia bastante llamativa en términos de todas las habilidades blandas sometidas a análisis, demostrando así la importancia de la integralidad del profesional como un elemento prioritario en el ejercicio profesional, tema consecuente con lo indicado por González y Villamil (2013, p. 3), quienes señalan que el ingeniero debe buscar el:

[...] método más de impartir o adquirir los conocimientos, actitudes, valores, destrezas y habilidades que permitan alcanzar los objetivos de conseguir las competencias requeridas, de acuerdo con el perfil profesional y lograr una formación integral, añadiendo a las competencias la inculcación de principios, éticas, valores para la correcta aplicación de las competencias en beneficio de la sociedad.

En términos más específicos sobresalen tres habilidades de mayor importancia, siendo estas el análisis y la resolución de problemas, el cumplimiento de objetivos y la adaptación al cambio. Puede señalarse, particularmente para la primera de ellas, que parece haber un ligamen al aspecto técnico y racional de las labores propias de la carrera profesional (Gonzalez y Villamil, 2013), esto pues denotaría el uso de pensa- 
miento analítico, así como de la creatividad para resolver posibles conflictos o situaciones que requieran un nivel de pensamiento más alto y estructurado. Habilidad, que, ligada a las otras dos, demuestra la importancia del cumplimiento efectivo de las tareas desarrolladas y una flexibilidad a nuevos paradigmas del conocimiento, así como del entorno (Guarin y Montoya, 2020), esto sin duda revela que el ingeniero ligado a las habilidades blandas debe ser analítico, preciso, efectivo, pero a la vez flexible, mismas que pueden ser definidas como Ejecutivas-Analíticas.

Puede determinarse un segundo grupo de habilidades blandas, que pueden ligarse más a la gestión de personal y grupos de trabajo, la cual menciona: “...habilidades de comunicación, inteligencia emocional, resolución de conflictos, negociación, influencia, desarrollo del espíritu de equipo y facilitación de grupos" (Barato, 2017, p. 503). Observando acá los aspectos de aprendizaje y desarrollo personal, trabajo en equipo y liderazgo. Puede determinarse que este segundo grupo de habilidades blandas, más allá de enfocarse en la ejecución de aspectos ligados a la ciencia o la técnica propias de la carrera, son dadas más hacia la integración social y personal, es decir, más hacia el aspecto humanista y sociológico del ejercicio profesional (Coser, 2019), temas que denotan una importante arista en términos de la formación y ejercicio profesional que los ingenieros deben tener en su vida laboral, esto pues, aunque se trate de carrera técnicas por esencia, esto no implica una inexistencia de contacto con otros individuos en sus trabajos. Estas pueden ser definidas como Crecimiento-Emocionales.

Por último, puede indicarse la existencia de un tercer grupo o juego de habilidades blandas, dadas más con un enfoque de comportamiento conductual o actitudinal de la persona (Guarin y Montoya, 2020), es decir, en términos del establecimiento de relaciones y ligámenes tanto con la técnica y materia específica, así como con otras personas, siendo estas la socialización, la lectura, y la deducción. Aspectos que pueden entenderse como los pilares en la actualización continua y el desarrollo constante del pensamiento crítico, pues en esencia, las tres combinan la capacidad de generar aprendizajes e interpretaciones de interés para el desarrollo de la carrera. Este último grupo pude definirse como las Socio-Interpretativas.

La siguiente figura detalla el esquema en términos de la jerarquía de importancia que parece existir en materia de las habilidades blandas en el ejercicio de la profesión ingenieril. 
Figura 5: Jerarquía de las habilidades blandas en la profesión ingenieril en Costa Rica, 2020-2021

\section{Ejecutivas - Analíticas}

- Análisis y la resolución de problemas

- Cumplimiento de objetivos

- Adaptación al cambio

\section{Crecimiento- Emocionales}

- Aprendizaje y desarrollo personal

- Trabajo en equipo

- Liderazgo

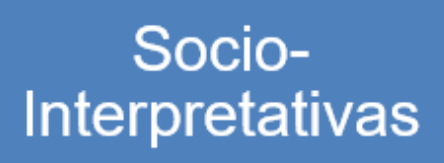

- Socialización

- Lectura

- Deducción

Fuente: Elaboración propia.

Al definirse estos tres tipos de niveles o grupos de habilidades blandas en el ejercicio de la profesión ingenieril, puede a su vez hacerse un ligamen al tipo de tecnología, o al menos al requerimiento específico que pueden potenciarlas, pudiendo definir así que las habilidades Ejecutivas-Analíticas parecen ligarse a aplicaciones y sistemas que impliquen la toma decisiones, la gestión de la información y la presentación de datos (Anderson et al., 2008), pues básicamente lo que buscan la solución eficaz de problemas.

Para el grupo de las de Crecimiento-Emocionales, pudiese darse un mayor ligamen a los sistemas de autoformación tales como los entornos de aprendizaje (Martí-Lobo, 2015) o bien incluso la inteligencia artificial, pues básicamente se busca el crecimiento y aprendizaje como persona.

Para el último grupo, las Socio-Interpretativas, destaca una mezcla de interés, pues se detalla el elemento social, así como la interpretación y análisis de contenidos, pudiendo observarse temas como la ingeniería de datos, los entornos sociales y la gestión de redes (Anderson, 2008), temas que tienen una mezcla de interés para este tipo de habilidades.

\section{Habilidades técnicas}

Los resultados hallados en referencia a las habilidades técnicas para el ejercicio profesional de la Ingeniería en el país son presentados en la figura anterior, en la cual, aunque pueden observarse algunos picos tendenciales en cuanto a resultados, sin duda logra evidenciarse, al igual que con las habilidades blandas, un balance de interés, denotando nuevamente la importancia de la integralidad intelectual y técnica como profesional (Tacchino, 2016). 
Figura 6. Habilidad técnica más importante, muestra de ingenieros, 2020-2021

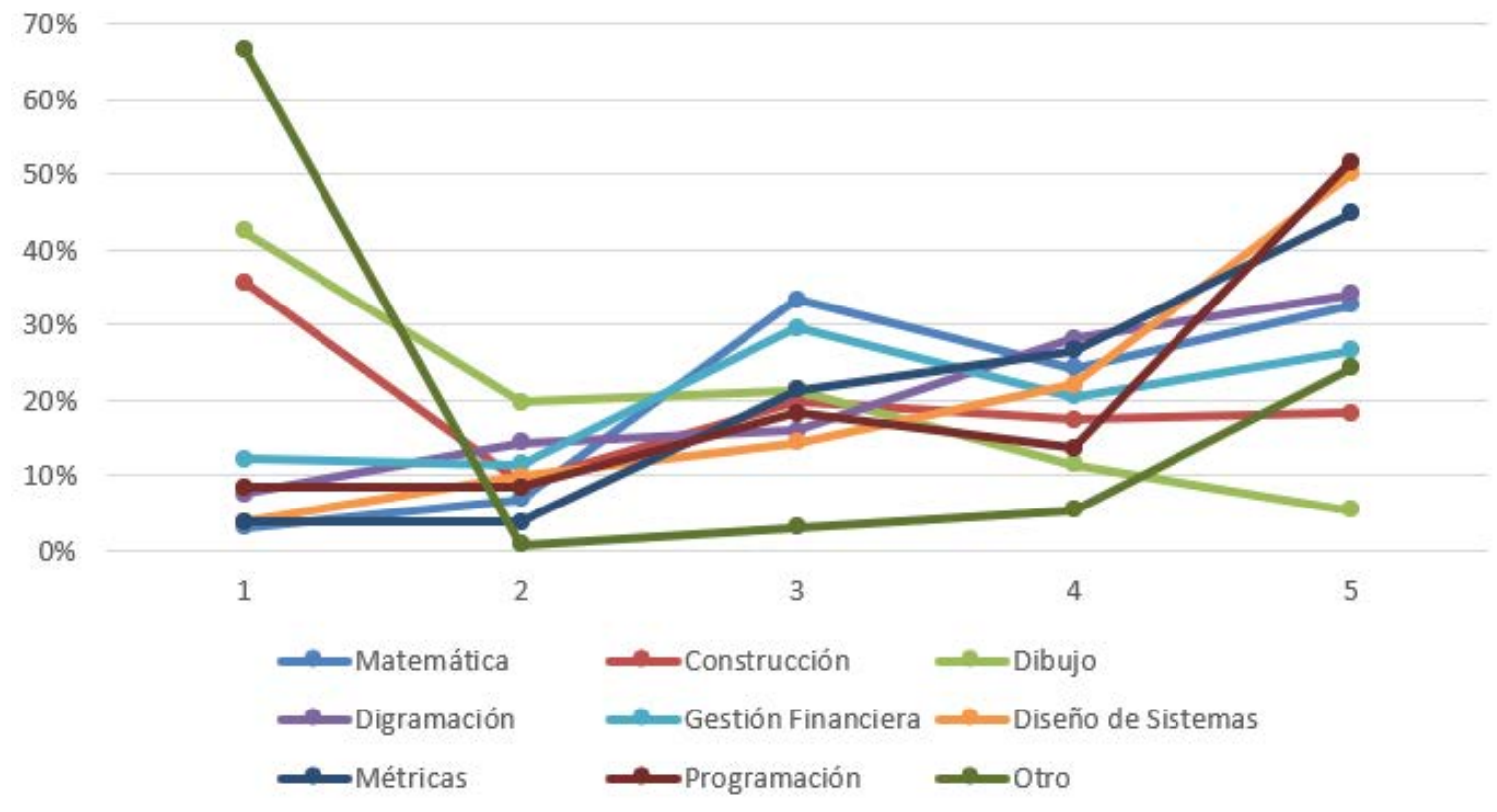

Fuente: Elaboración propia con base en trabajo de campo.

Resulta de interés acá precisar el concepto de habilidad técnica, también entendida como habilidad dura, la cual según Worboys et al. (2019, p. 268):

Son las capacidades requeridas para profesiones específicas generalmente relacionadas con las herramientas, técnicas o conocimientos profesionales que nos permiten trabajar dentro de nuestra profesión. Estas no solo son fáciles de observar, cuantificar y medir, también suelen ser fáciles de enseñar cuando son nuevas para el alumno y donde no es necesario "desaprender".

$\mathrm{Al}$ analizar estas habilidades en la profesión ingenieril en el país, puede observarse la evidente relevancia de dos de ellas, siendo la programación y el diseño de sistemas, las cuales radican principalmente en temas ligados a la creación y al desarrollo de soluciones tecnológicas que permitan maximizar la efectividad de procesos (Font, 2011), ideas que parecen estar bastante ligadas a las habilidades blandas dada por la solución de problemas, denotando así un ligamen de interés entre los dos grupos de habilidades en importancia, entiéndase las antes señaladas como técnicas, así como las Analíticas-Ejecutivas.

Adicionalmente puede indicarse que la matemática como tal, y el uso y desarrollo de métricas, sin duda son aún, habilidades que por definición misma, son parte de la profesión, claro está, dadas por un ligamen técnico y un ejercicio particular del sistema neural ligado al córtex que genera el pensamiento analítico y matemático, tal cual se observa en lo expresado por Márquez (2020, p. 33), quien indica que: “...es la parte más desarrollada de nuestro encéfalo podríamos decir que es la adquisición más novedosa y actualizadas que tenemos, es funcional, lógico y analítico". 
De forma clara entonces, puede asociarse que la Ingeniería, indiferentemente de su área de especialidad, puede aún definirse como una ciencia exacta y ligada a la técnica matemática, la cual requiere un grado de pensamiento analítico de relevancia, aspecto que también es observable de forma extrapolada en las habilidades blandas y en los requerimientos tecnológicos que estas tienen, particularmente en los segundos grupos de estas, dados por el auto aprendizaje y la deducción específicamente.

Una habilidad técnica de interés que resalta, pero con una valoración media, es la gestión financiera, evaluación que no dejar de ser curiosa, pues indiferentemente del área de ejercicio, el manejo financiero es de importancia, entiéndase para efectos empresariales o personales, más sin embargo esta no denota la mayor relevancia del caso. Esta habilidad puede ser ligada de forma directa a las de Crecimiento-Emocionales en términos de las habilidades blandas antes detalladas, pues en esencia hace mención del manejo y proyección de los recursos monetarios en aras de maximizar la gestión personal o bien de una institución, temas relacionados incluso con la conducta misma de la persona (Martí-Lobo, 2015).

Finalmente puede señalarse que las dos habilidades técnicas que parecen tener una menor evaluación, más no por ello menos importantes en el desarrollo de la profesión, son las dadas para las habilidades más ligadas a la parte motora, es decir con ligamen al córtex motor e incluso el lóbulo parietal (Kolb y Whishaw, 2008).

Lo anterior demuestra una estrecha relación de las habilidades ligadas a la creación motriz y la capacidad de generar entregables en las tareas a desarrollar, es decir a la generalización de resultados a partir de acciones específicas, tema que puede a su vez ser ligado a la habilidad blanda de la deducción, que básicamente parte de la idea de permitir llegar a cuestiones e ideas específicas a partir de generalidades, lo que parece denotar que la conducta investigativa estar implícita en la personalidad del ingeniero, pero que encuentra su momento de evidencia en estas habilidades técnicas, que en efecto, aunque en importancia no parecen ser las más relevantes en el ejercicio de la profesión como tal. Es importante señalar acá que las habilidades técnicas, aunque puede ser analizadas en ligamen a las blandas, en función de los perfiles de conducta de los profesionales, así como sus experiencias previas (Cowan, 2006), representan por sí mismas las características propias en materia cognitiva y práctica que un profesional debe tener, siendo en este caso el ingeniero.

Para finalizar el análisis de las habilidades técnicas, cabe resaltar, y con base en los resultados obtenidos, la importancia de considerar la formación propia de la ingeniería como un tema sistémico e integral, en la que, a pesar de denotarse algunas áreas de mayor relevancia, no deben por sí mismas, descuidarse otras, pues la maximización del conocimiento debe ser dado por la integración de diferentes técnicas y áreas epistemológica, logrando formar así un profesional polifacético e integral.

Como un dato adicional se precisa la posible discriminación existente en el ejercicio de la profesión ingenieril en el país, Señalando resultados bastante similares en cuanto a las respuestas afirmativas y negativas, con una breve ventaja para aquellos profesionales que señalan que si se presenta la discriminación en la carrera. 
Figura 7. Discriminación en la profesión ingenieril, muestra de ingenieros, 2020-2021

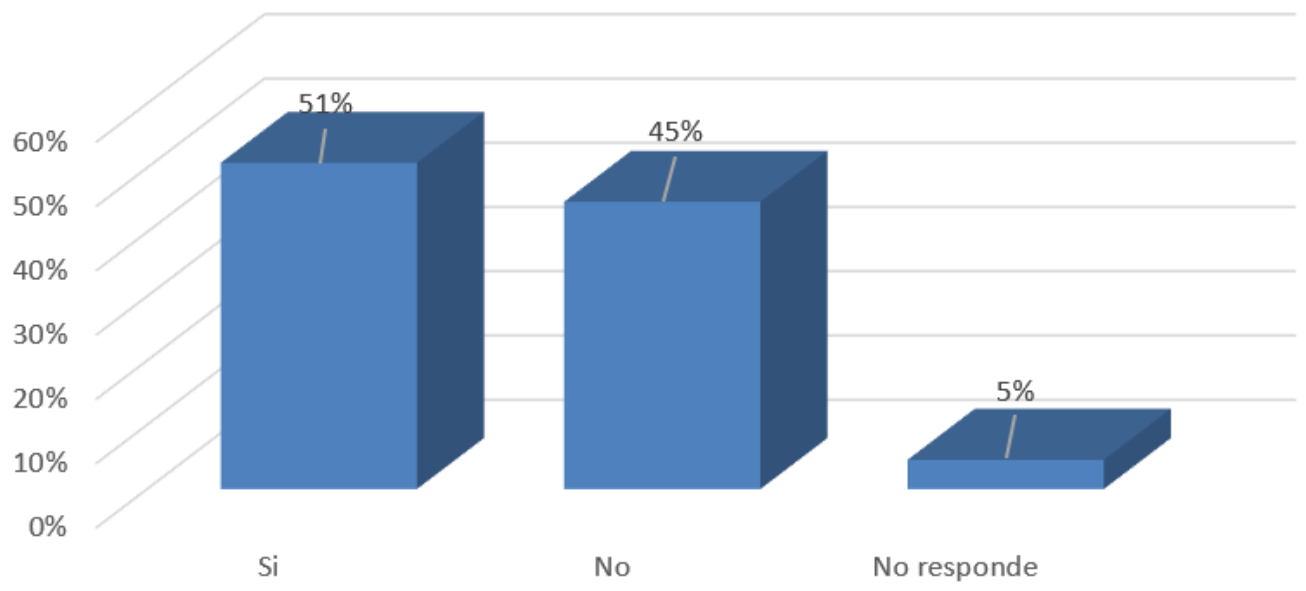

Fuente: Elaboración propia con base en trabajo de campo.

En este punto es de interés señalar que la discriminación es aquella que se da cuando, de acuerdo con Currea (2007, p.114):

[...] los procesos de selección laboral responden más a las reglas no escritas, independientes de la productividad del trabajador, que se basan en las experiencias previas o en el desagrado subjetivo por parte de oferentes y demandantes por un determinado grupo social.

Observándose que esta puede ser dada por cualquier situación o hecho que afecte el desarrollo digno de la profesión. Precisamente las causas de mayor discriminación en la carrea ingenieril, son presentadas en la siguiente figura, esto con base en las respuestas afirmativas, es decir considerando únicamente aquellos profesionales que consideran la existencia de algún tipo de discriminación en la carrera. 
Figura 8. Razón de discriminación en el ejercicio de la profesión, muestra de ingenieros, 2020-2021

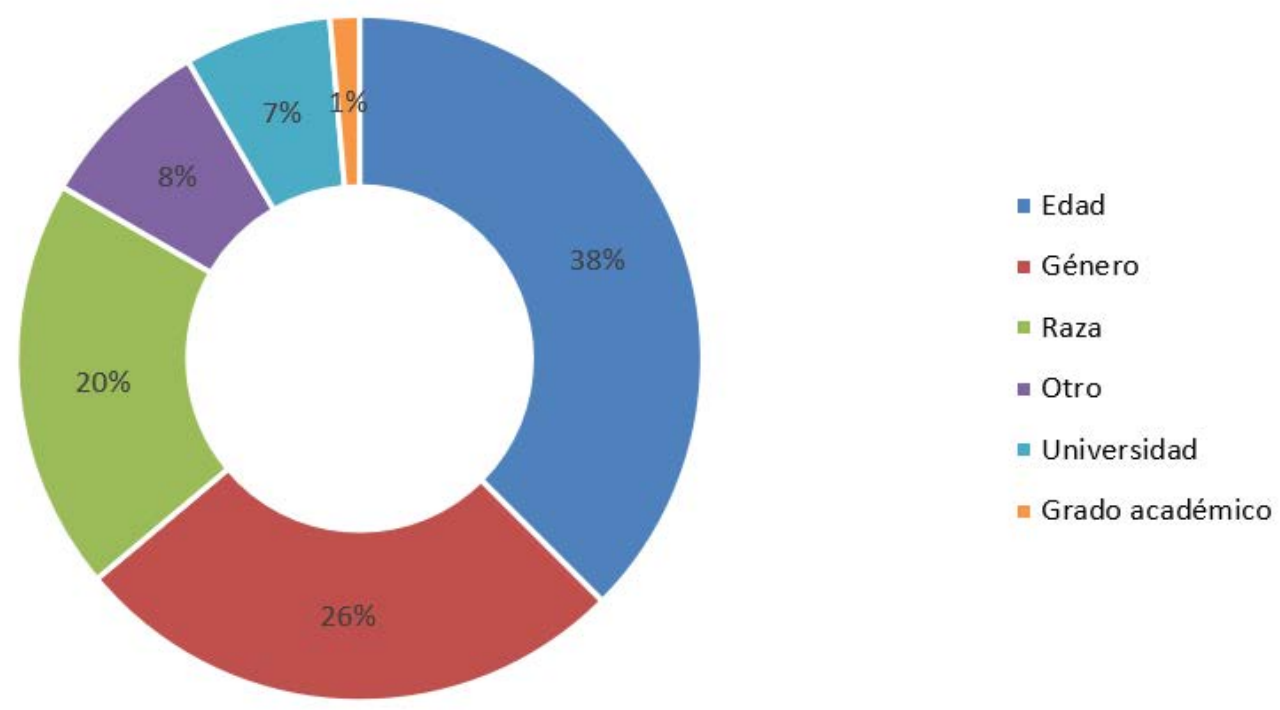

Fuente: Elaboración propia con base en trabajo de campo.

Puede observarse como primera causa de discriminación en el ejercicio profesional de la ingeniería la edad, factor que puede ser asociado a una percepción y precognición derivada de la idea de que a mayor edad se tiene más experiencia (Renvoisé y Morin, 2010). Seguido por el género, variable que suele ser observable en temas de discriminación laboral. Mientras que la tercera causa es dada por la raza, siendo los tres elementos de tipo demográfico, en donde parece analizarse a la persona como tal, más no a las habilidades técnicas o blandas del profesional en cuestión.

Es de interés señalar que en la opción de otros se señalan aspectos como la apariencia física y el estado civil, variables que responden de igual forma a aspectos perceptivos más que objetivos (Coser, 2019), que no debieran interferir con las habilidades propias del ingeniero.

Por último cabe indicar que se señala la universidad de procedencia como un factor de discriminación, aspecto que pareciera en primera instancia ser de corte valorativo y subjetivo (individual), pues claramente la universidad cumple el rol de formación, más no del ejercicio de la profesión como tal, no obstante si esta valoración discriminativa es hecha con base en las funciones que la persona desarrolla en relación a su formación universitaria, sí podría existir una especie de "discriminación objetiva", pues se estaría haciendo en función de las habilidades técnicas obtenidas en la institución en cuestión.

Sin duda puede implicarse que la discriminación en la profesión parece ser dada más por variables perceptivas ligadas a precogniciones sociales (Umaña y Vargas, 2018), es decir a ideas preconcebidas, que no necesariamente son correctas, y que claramente menoscaban el ejercicio digno de la profesión ingenieril en términos prácticos. 


\section{Análisis comparativo de habilidades y tecnologías}

Una vez completado el análisis de las principales habilidades en el desarrollo de la profesión ingenieril, tanto desde un enfoque técnico, así como aquellas denominadas blandas, procede a realizarse una especie de exegesis en relación con las aplicaciones y requerimientos en tecnologías, y en función del cruce entre los diferentes grupos de habilidades ambos tipos encontradas.

Tabla 1. Habilidades blancas y técnicas, y su relación con tecnologías en la profesión ingenieril

\begin{tabular}{|c|c|c|c|c|c|c|c|}
\hline \multicolumn{8}{|c|}{ Habilidad técnica } \\
\hline \multirow{3}{*}{$\begin{array}{l}\text { Jerarqúía } \\
\text { de habi- } \\
\text { lidades } \\
\text { blandas }\end{array}$} & & $\begin{array}{l}\text { Programación } \\
\text { y desarrollo de } \\
\text { sistemas }\end{array}$ & $\begin{array}{l}\text { Desarrollo y uso } \\
\text { de métricas }\end{array}$ & $\begin{array}{l}\text { Gestión } \\
\text { financiera }\end{array}$ & $\begin{array}{l}\text { Habili- } \\
\text { dades } \\
\text { motoras }\end{array}$ & & \multirow[b]{2}{*}{$\begin{array}{l}\text { Habilidad } \\
\text { blanda }\end{array}$} \\
\hline & $\begin{array}{l}\text { Ejecuti- } \\
\text { vas-Aná- } \\
\text { liticas }\end{array}$ & $\begin{array}{l}\text { Programación } \\
\text { matemática, sis- } \\
\text { temas inteligen- } \\
\text { tes para la toma } \\
\text { de decisiones }\end{array}$ & $\begin{array}{l}\text { Métricas de con- } \\
\text { trol y resultados }\end{array}$ & $\begin{array}{l}\text { Simulación } \\
\text { financiera } \\
\text { y toma de } \\
\text { decisiones }\end{array}$ & $\begin{array}{l}\text { Aplica- } \\
\text { ciones de } \\
\text { esquema- } \\
\text { tización y } \\
\text { resumen } \\
\text { de textos }\end{array}$ & $\begin{array}{c}\text { Buena escu- } \\
\text { cha }\end{array}$ & \\
\hline & $\begin{array}{l}\text { Creci- } \\
\text { miento- } \\
\text { Emocio- } \\
\text { nales }\end{array}$ & $\begin{array}{l}\text { Tecnologías de } \\
\text { vídeo, Gestión } \\
\text { de Juegos }\end{array}$ & $\begin{array}{l}\text { Evaluación del } \\
\text { desempeño y } \\
\text { gestión de la } \\
\text { evaluación de la } \\
\text { conducta }\end{array}$ & $\begin{array}{l}\text { Juegos de } \\
\text { inversión y } \\
\text { capacitación }\end{array}$ & $\begin{array}{l}\text { Tecnolo- } \\
\text { gías de } \\
\text { comuni- } \\
\text { cación } \\
\text { social }\end{array}$ & $\begin{array}{l}\text { Conciliador } \\
\text { e integra- } \\
\text { dor }\end{array}$ & \\
\hline & $\begin{array}{l}\text { Socio- } \\
\text { Interpre- } \\
\text { tativas }\end{array}$ & $\begin{array}{l}\text { Inteligencia } \\
\text { artificial para la } \\
\text { toma de deci- } \\
\text { siones }\end{array}$ & $\begin{array}{c}\text { Sistemas de } \\
\text { autoevaluación } \\
\text { y gestión de } \\
\text { personal }\end{array}$ & $\begin{array}{l}\text { Análisis de } \\
\text { casos de } \\
\text { estudio en } \\
\text { términos } \\
\text { financieros }\end{array}$ & $\begin{array}{c}\text { Sistemas } \\
\text { de diseño } \\
\text { gráfico y } \\
\text { presen- } \\
\text { tación de } \\
\text { resulta- } \\
\text { dos }\end{array}$ & $\begin{array}{l}\text { Comunica- } \\
\text { ción }\end{array}$ & \\
\hline
\end{tabular}

Fuente: Elaboración propia con base en trabajo de campo.

En la tabla anterior puede observarse el cruce detallado para las habilidades técnicas de mayor relevancia encontradas en el estudio, así como las habilidades blandas correspondientes, las cuales a su vez son precisadas en una correlación directa (Alvarado y Santiesteban, 2011) con la jerarquía de las habilidades blandas previamente definidas. El detalle presentado en cada cuadrante a lo interno de la tabla detalla la tecnología o bien el enfoque en el requerimiento que parece ser el más indicado en función del cruce de habilidades precisado.

Puede observarse que a medida que el requerimiento se ubica más hacia el extremo superior izquierdo, se detalla una precisión mayor de habilidades técnicas con un enfoque directo a la capacidad analítica y de resolución de problemas, incluso ligado a habilidades matemáticas (Font, 2011), es decir, quizás un poco 
más ligadas a la gestión técnica propiamente de la ingeniería. Por otra parte, a medida que estas tecnologías se acercan al cuadrante inferior derecho, resaltan los elemento sociales y emocionales de los profesionales (Sánchez, 2020), enfocándose en aspectos más ligados a la gestión gráfica y social propiamente.

Las tecnologías y sus requerimientos específicos ubicados en parte izquierda e inferior detonan una mezcla de interés entre los elementos sociales, comunicativos, y de análisis (Rimé, 2012), mientras que los ubicados a la derecha y arriba, pueden observarse habilidades que combinan aspectos ligados a un enfoque de análisis, pero más ligados a la ejecución y práctica, sujetadas a su vez a las habilidades motoras.

Claramente cada tecnología y su requerimiento específico debe ser analizado de forma individual, esto pues el desarrollo de sistemas o aplicaciones debe responder a los requerimientos básicos de cada situación en particular, sin embargo, la tabla anterior, detallar un posible modelo de potencialización de habilidades ingenieriles en función de una tecnología en particular.

\section{Conclusiones}

Las habilidades blandas de mayor relevancia en el ejercicio de la profesión ingenieril son dadas en función de la buena escucha, la conciliación y la comunicación, elementos que denotan ser extrapolables a sistemas que recopilen, analices y presenten información de forma efectiva, lo que señala que las aplicaciones y sistemas informáticos y operativos que precisen esta relación parecieran beneficiar el potencial de las habilidades blandas en la profesión ingenieril.

Puede concluirse que el perfil conductual y de requerimientos ingenieriles en términos de las habilidades blandas demandadas para el ejercicio profesional, son dados en tres grupos, con una aparente jerarquía de importancia, siendo estos, el primero las habilidades Ejecutivas-Analíticas, el segundo las habilidades de Crecimiento-Emocionales, y las últimas las Socio-Interpretativas. Resaltando la importancia de la integralidad como un elemento fundamental en la gestión de profesional y personal del ingeniero como un profesional y en ligamen directo a sus habilidades blandas.

Existe un ligamen entre los grupos de habilidades blandas encontrados y los tipos de tecnologías o aplicaciones que ayuden a potenciarlas, incluyendo los sistemas de toma de decisiones para el grupo de habilidades Analíticas-Ejecutivas, aplicaciones e autoaprendizaje e inteligencia artificial para las de Crecimiento-Emocionales, y tecnologías ligadas a la ingeniería de datos y la gestión de redes para las habilidades Socio-Interpretativas, tema que permite determinar posibles requerimientos para efectos del desarrollo de tecnologías en estas áreas.

Las habilidades técnicas o "duras" de mayor relevancia para el desarrollo de la profesión ingenieril hacen referencia a la programación y el diseño de sistemas en primera instancia, seguido por las métricas y su uso, elementos que demuestran una capacidad analítica implícita, tanto en la interpretación de información, así como en el desarrollo del pensamiento creativo para brindar soluciones de relevancia y valor en la profesión, aspectos a que a su vez tienen un ligamen directo a las habilidades blandas de mayor importancia, las cuales son dadas por al análisis y la solución de problemas.

La discriminación en el ejercicio profesional de la ingeniería es una variable que afecta su correcto desarrollo, observándose que en más de la mitad de los casos analizados se dan este tipo de afectaciones, siendo las variables demográficas de la persona, y basadas en percepciones preconcebidas y de corte subjetivo e interpretativo, las que tienden a darse en términos discriminatorios, tales como la edad, el 
género y otros. Por lo que se señala que las habilidades técnicas no son un factor de peso en términos de la discriminación ingenieril, esto pues, aunque la universidad de procedencia también denota tener resultados de interés, parecen ser dados más por un tema de interpretación subjetiva que por un aspecto ligado al ejercicio técnico como tal.

Al realizar un cruce entre las habilidades blandas y técnicas, con base un análisis de los requerimientos propios de la tecnología que pudiese potenciar esas habilidades, logra observarse una relación de interés entre la solución de problemas y los sistemas complejos de toma decisiones, mientras que para las habilidades sociales, parece haber un mayor ligamen a tecnologías ligadas a la generación de comunicación, el estudio de casos y la participación en dinámicas, señalando a la vez posibles relaciones de importancia entre las habilidades blandas y técnicas en el uso de tecnologías en la profesión ingenieril.

$\mathrm{Al}$ analizar los datos, y al validar estos con el coeficiente de Cronbach, así como al aplicar los análisis cruzados de las variables, se observa una consecución precisa del objetivo general, pudiendo señalar las habilidades técnicas de mayor relevancia en el ejercicio de la profesión ingenieril en Costa Rica, además de lograr plantear las bases para un modelo de análisis de las habilidades en cuestión y su aplicación en el campo laboral de la ingeniería.

\section{Estudios futuros}

Una vez expuestos los resultados, el análisis, las propuestas y las conclusiones del trabajo, se señala que los hallazgos evidenciados en este documento dan paso al desarrollo de futuros estudios ligados al campo de las habilidades blandas y técnicas en el desarrollo profesional de la Ingeniería. Entre estos, destacan la eventual correlación que pudiese existir entre las habilidades blandas y técnicas, esto en aras de precisar los cruces de mayor relevancia en materia aplicativa laboral. Adicionalmente, se da paso a la prueba empírica y la aplicación de modelo de agrupamiento de las habilidades analizadas en este documento y su precisión en las categorías antes señaladas, tema que pudiese ser probado en la práctica, logrando a su vez definir mejores métodos de gestión del recurso humano en el ejercicio de la profesión ingenieril.

\section{Bibiliografía}

Alonso, F., Martínez, M. y Segovia, J. (2005). Introducción a la ingeniería del software: Modelos de desarrollo de programas. Delta Publicaciones.

Anderson, J. (2008). Redes neurales. Alfaomega.

Anderson, D., Sweeney, D., y Williams, T. (2008). Estadística para Administración y Economía (10 $10^{a}$ Ed.). Cengage Learning.

Alvarado, J. y Santiesteban, C. (2011). La validez en la medición psicológica. UNED Publicaciones.

Barato, J. (2017). El director de proyectos a examen. Guía de estudio en español para la capacitación del directos de proyectos. Editorial Díaz de Santos.

Bermúdez, J., Pérez, A., Ruíz, J., Suárez, P. y Rueda, B. (2012). Psicología de la personalidad. Universidad Nacional de Educación a Distancia. Recuperado de: http://hdl.handle. net/20.500.12226/603. 
Blanco, A. (2009). Desarrollo y evaluación de competencias en Educación Superior. Narcea S. A. de Ediciones.

Cowan, L. (2006). Edges of Experience: Memory and Emergence: Proceedings of the 16th international IAAP congress for analytical psychology. Daimon.

Coser, L. (2019). Maestros del pensamiento sociológico. Las ideas en su contexto histórico y social. Centro de Investigaciones Sociológicas.

Crisman, R. (2016). La construcción de escalas de medición para la investigación lingüística y sus aplicaciones didácticas. Una propuesta con respecto a la modalidad lingüista andaluza. ACCI Ediciones.

Currea, C. C. (2007). Estudios de género en el DANE. Pontificia Universidad Javeriana.

Font, B. (2011). Programación matemática para la economía y la empresa (2ª ed.). Universitat de Valencia.

Galera, E. (2015). Relación entre inteligencias múltiples, creatividad y rendimiento académico en matemáticas. AntropiQa.

González, O. y Villamil, M. (2013). Introducción a la ingeniería. Una perspectiva desde el currículo en la formación del ingeniero. Ediciones Ecoe.

Guarin, C. y Montoya, O. (2020). Fisioterapia en salud mental. Editorial Universidad del Rosario.

Kolb, B. y Whishaw, I. (2008). Neuropsicología humana (5ª Ed.). Editorial Médica Panamericana.

Luna, F., Peña, C. y Iacano, M. (2018). Programado web full stack. Desarrollo frontend y backend. Buenas prácticas: análisis, test y optimización. Redusers.

Márquez, A. (2020). Principios neurológicos y psicológicos de la inteligencia emocional. Centro de Capacitaciones e Innovación Educativa.

Martí-Lobo, P. (2015). Procesos y programas de neuropsicología educativa. Secretaría General Técnica. Centro de Publicaciones. Ministerio de Educación, Cultura y Deporte.

Navarro, J. (2011). Epistemología y metodología. Grupo editorial Patria.

Renvoisé, P. y Morin, C. (2010). Neuromarketing. El nervio de la venta. Editorial UOC.

Rimé, B. (2012). La compartición social de las emociones. Editorial Desclée de Brouwer, S. A.

Sánchez, J. (2020). Perfiles neuro-conductuales en la ingeniería en Costa Rica: Propuesta de modelo teórico y neuro-inductivo de perfiles profesionales. Revista Nuevo Humanismo, 8(2), 30. DOI: https://doi.org/10.15359/rnh.8-2.3.

Solomon, M. (2013). Comportamiento del consumidor (10 ${ }^{\text {a }}$ Ed.). Pearson.

Tacchino, A. (2016). Perfil profesional del ingeniero industrial y comercial en una universidad privada: percepciones de académicos y empleadores (Tesis doctoral). Universidad San Ignacio de Loyola. Recuperado de: http://repositorio.usil.edu.pe/handle/USIL/1923.

Tovar, R. (2012). Habilidades gerenciales. Desarrollo de destrezas, competencias y actitud. Ecoe Ediciones.

Umaña, P. y Vargas, L. (2018). La participación de las mujeres continúa relegada en las ciencias. Noticias UCR, 10.

Worboys, G., Lockwood, M. y Kothari, A. (2019). Gobernanza y gestión de áreas protegidas. Editorial Universidad del Bosque. 